\title{
Nanoscale
}

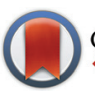

CrossMark \& click for updates

Cite this: Nanoscale, 2015, 7, 9083

Received 30th September 2014 Accepted 14th April 2015

DOI: 10.1039/c4nr05742b

www.rsc.org/nanoscale

\section{RNAi-based glyconanoparticles trigger apoptotic pathways for in vitro and in vivo enhanced cancer-cell killing $\dagger$}

\author{
João Conde,,$^{\mathrm{a}}$ Furong Tian, $\xi^{\mathrm{b}}$ Yulan Hernandez, ${ }^{\mathrm{c}}$ Chenchen Bao, ${ }^{\mathrm{d}}$ \\ Pedro V. Baptista, ${ }^{e}$ Daxiang Cui, ${ }^{d}$ Tobias Stoeger $^{\star b}$ and Jesus M. de la Fuente*c,d,f
}

Gold glyconanoparticles (GlycoNPs) are full of promise in areas like biomedicine, biotechnology and materials science due to their amazing physical, chemical and biological properties. Here, siRNA GlycoNPs (AuNP@PEG@Glucose@asiRNA) in comparison with PEGylated GlycoNPs (AuNP@PEG@Glucose) were applied in vitro to a luciferase-CMT/167 adenocarcinoma cancer cell line and in vivo via intratracheal instillation directly into the lungs of B6 albino mice grafted with luciferase-CMT/167 adenocarcinoma cells. siRNA GlycoNPs but not PEGylated GlycoNPs induced the expression of pro-apoptotic proteins such as Fas/CD95 and caspases 3 and 9 in CMT/167 adenocarcinoma cells in a dose dependent manner, independent of the inflammatory response, evaluated by bronchoalveolar lavage cell counting. Moreover, in vivo pulmonary delivered siRNA GlycoNPs were capable of targeting c-Myc gene expression (a crucial regulator of cell proliferation and apoptosis) via in vivo RNAi in tumour tissue, leading to an $~ 80 \%$ reduction in tumour size without associated inflammation.

\section{Introduction}

Carbohydrates are, together with nucleic acids and proteins, important molecules for life. Although individual carbohydrate interactions are relatively weak, nature utilizes multivalent interactions between these cell surface ligands and their respective biological receptors to modulate biological events such as the ones related to cell adhesion, normal tissue growth and repair, viral/bacterial infection, signal transduction, trap-

\footnotetext{
${ }^{a}$ Massachusetts Institute of Technology, Institute for Medical Engineering and Science, Harvard-MIT Division for Health Sciences and Technology,

E25-449 Cambridge, Massachusetts, USA

${ }^{b}$ Comprehensive Pneumology Centre, Institute of Lung Biology and Disease, Helmholtz Zentrum München, Neuherberg, Germany.

E-mail: tobias.stoeger@helmholtz-muenchen.de

${ }^{c}$ Instituto de Nanociencia de Aragon (INA), Universidad de Zaragoza, Zaragoza, 50018, Spain. E-mail: jmfuente@unizar.es

${ }^{d}$ Institute of Nano Biomedicine and Engineering, Key Laboratory for Thin Film and Microfabrication Technology of the Ministry of Education, Research Institute of Translation Medicine, Shanghai Jiao Tong University, Dongchuan Road 800, 200240 Shanghai, People's Republic of China

${ }^{e}$ UCIBIO, Departamento de Ciências da Vida, Facldade de Ciências e Tecnologia, Universidade Nova de Lisboa, Campus de Caparica, 2829-516 Caparica, Portugal

${ }^{f}$ Instituto de Ciencia de Materiales de Aragón-CSIC/Universidad de Zaragoza, Spain $\dagger$ Electronic supplementary information (ESI) available: Synthesis, functionalization and quantification methods for siRNA-gold glyconanoparticles. See DOI 10.1039/c4nr05742b

$\$$ Both authors have contributed equally to this manuscript.
}

ping of leukocytes, and gene transfer. So the decoding of carbohydrate interactions opens up the possibility to employ physiologically inert gold nanoclusters in diagnostics and/or therapy. ${ }^{1}$ Among them, gold glyconanoparticles (GlycoNPs) have drawn more attention owing to their well-defined features as water-soluble carbohydrate-functionalized nanoclusters with promising potential for chemical glycobiology, biomedicine, diagnostics and clinical applications. In the last 10 years, de la Fuente and co-workers have extensively reported a pioneering integrated glyconanotechnology strategy. Based on the use of these nanoparticles they studied and evaluated carbohydratecarbohydrate, and carbohydrate-protein interactions, ${ }^{2-6}$ and further used these nanoparticles as potential tools in antiadhesive therapy, ${ }^{7}$ for cell-cell adhesion studies ${ }^{8}$ and for the prevention of pathogen invasion. ${ }^{9}$

Small carbohydrates such as glucose can be attached to gold nanoparticles (AuNPs) and thereby used for sensitive colorimetric assays. ${ }^{10}$ However, to the best of our knowledge the use of siRNA GlycoNPs in the regulation of important genes of the apoptotic pathways has never been described.

Herein, a novel structure of multifunctional RNAi-based GlycoNPs functionalized with glucose, poly(ethylene glycol) (PEG), a biotin linked fluorophore and cMyc targeting siRNA was designed to trigger apoptosis and gene silencing pathways (see Fig. 1). Through a chemical approach, the functional properties and moieties of this kind of multifunctional nanostructure can be easily tuned and quantified as recently 


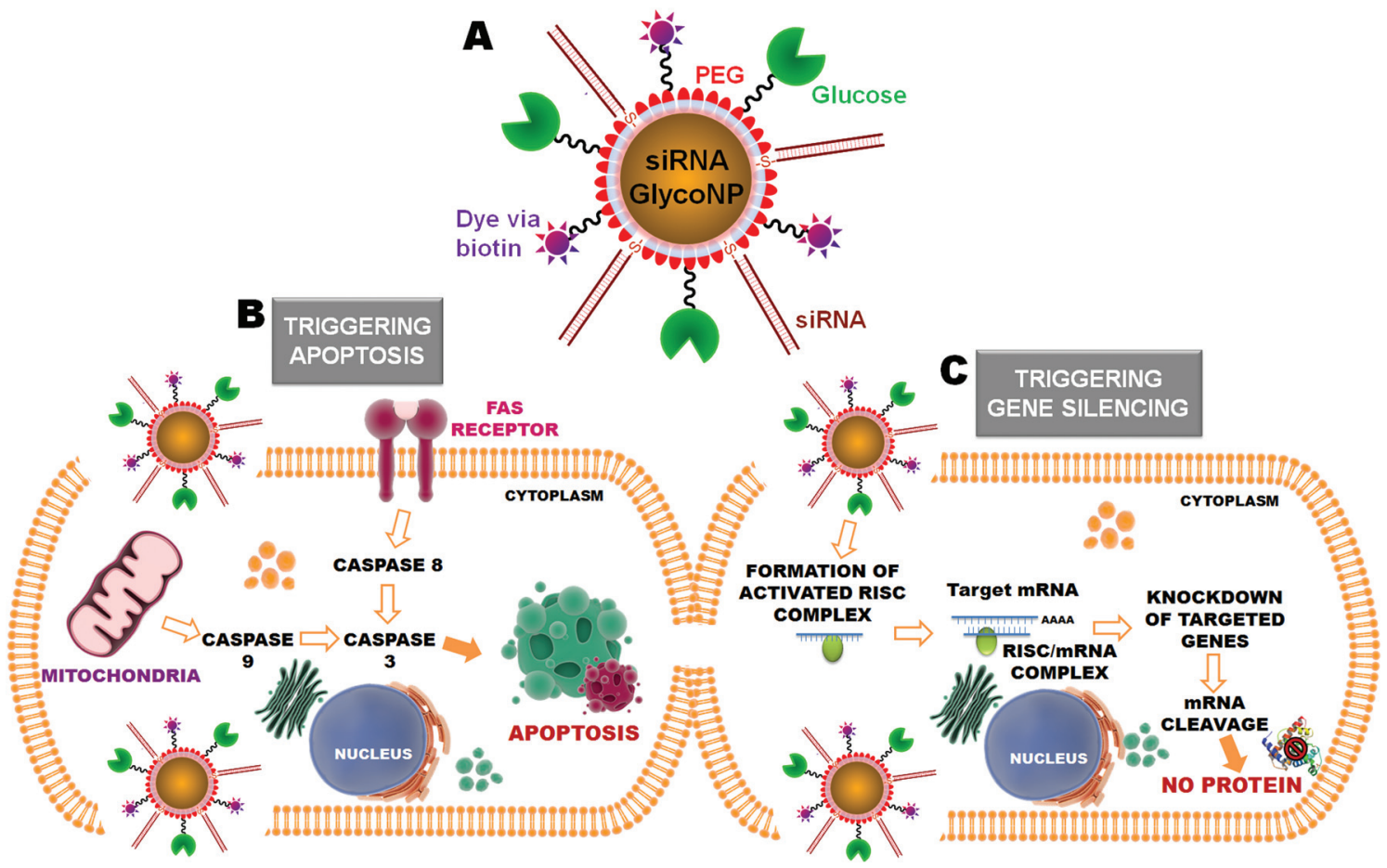

Fig. 1 (A) Multifunctional siRNA glyconanoparticles (siRNA GlycoNPs) trigger apoptotic pathways (B) with the expression of cell death receptors (Fas) and caspases. The death domain-containing receptor Fas can sense an external signal and activate the apoptosis pathway through the Fasrelated death domain. This pathway is mediated by the activation of caspase-8, followed by direct cleavage of downstream effector caspases. The apoptosis pathway can also be initiated in the cytoplasm through activation of intracellular changes resulting in the release of proapoptotic factors from the mitochondria. The release of these factors leads to the activation of caspase- 9 , and ultimately results in the activation of effector caspases (e.g. caspase 3) and consequently to cell death by apoptosis. (C) The siRNA glycoNPs also have the capacity to trigger gene silencing via activation of the RNA interference pathway, by small interfering RNA (siRNA), promoting nucleolytic degradation of the target mRNA and/or translational suppression.

reported by our group. ${ }^{11,12}$ In fact, we have provided evidence of in vitro and in vivo efficient RNAi via the synthesis of a library of novel multifunctional AuNPs, tested in three biological systems of increasing complexity: in vitro cultured human cells (HeLa cells), in vivo freshwater polyp (Hydra vulgaris), and in vivo mice models (B6 albino mice). ${ }^{11,13}$

In view of the great number of studies concerning glucotoxicity attributed to the inability of cells to reduce glucose uptake when exposed to chronic hyperglycemia, we designed novel siRNA GlycoNPs that predispose gene expression to apoptosis via enhancement of cell death receptors. In fact, it is known that high levels of glucose induce apoptosis via upregulation of cell death receptors - Fas - providing a link between type 1 and type 2 diabetes. ${ }^{14,15}$ Hyperglycemia is the central initiating factor for all types of diabetic microvascular disease. Studies on endothelial cell cultures clearly show glucose toxicity by delaying replication, disturbing the cell cycle, increasing DNA damage and accelerating cell death. ${ }^{16,17}$

Maedler et al. also reported that increased glucose concentration by itself induces apoptosis in human pancreatic $\beta$-cells via the upregulation of Fas receptors, which can interact with the constitutively expressed FasL (Fas ligand) on neighbouring $\beta$-cells. Fas-FasL interaction leads to cleavage of procaspase- 8 to caspase-8, which promotes caspase-3 activation. ${ }^{14}$ The stimulation of death receptors such as Fas (APO-1/CD95) and creation of DISC (death-inducing signalling complex) are commonly considered as the starting point in the extrinsic apoptosis execution phase, whereas caspase- 9 activation is a downstream marker for mitochondrial membrane permeabilisation in the intrinsic apoptotic pathway. ${ }^{18}$ Both pathways converge on the same terminal outcome, which is initiated by the cleavage of caspase- 3 and results in DNA fragmentation, degradation of cytoskeletal and nuclear proteins, formation of apoptotic bodies, expression of ligands for phagocytic cell receptors and finally clearance by phagocytic cells.

Herein, the in vitro and in vivo activation of apoptotic and gene silencing pathways via RNAi GlycoNPs will be investigated and the clinical outcome in terms of lung cancer progression will be evaluated.

\section{Results and discussion}

\section{Glyconanoparticle synthesis and characterization}

Multifunctional gold glyconanoparticles were prepared by reduction of sodium tetrachloroaurate(III) hydrate with sodium citrate as described by Turkevich and Frens, ${ }^{19,20}$ and stabilized with poly(ethylene glycol) (PEG) and subsequent conjugation 
with glucose, biotin and siRNA. Briefly, the obtained AuNPs, with an average diameter of $14 \mathrm{~nm}$, were subsequently functionalized with two types of thiolated PEG - a commercial carboxylated PEG (HS-EG(8)- $\left(\mathrm{CH}_{2}\right)_{2}-\mathrm{COOH}$ ) and another one synthesized in our lab with an azide group at the end (HS- $\left.\left(\mathrm{CH}_{2}\right)_{3}-\mathrm{CONH}-\mathrm{EG}(6)-\left(\mathrm{CH}_{2}\right)_{2}-\mathrm{N}_{3}\right)$ - by exchange of the citrate groups. For the subsequent attachment of thiolated siRNA using the covalent approach previously described, ${ }^{11}$ the AuNPs were functionalized with a $40 \%$ degree of coverage of the surface using $50 \%$ of each PEG chain.

Once stable and biocompatible PEGylated AuNPs were obtained we functionalized them with amine-modified biotin and glucose through an EDC coupling reaction forming amide bonds with the carboxylic groups exposed on the surface. The covalent conjugation of the biotin was evaluated by the crosslinking induced by the effective attachment of streptavidin to up to four biotin molecules and also determined by indirect quantification by the Bradford assay. Concerning the attachment of glucose, the change of the net charge and the aggregation of the AuNPs effectively functionalized in the presence of the lectin Concanavalin A (Con A) ${ }^{21}$ were used to confirm the conjugation, again via the occurrence of crosslinking induced due to the multi-interaction between glucose and Con A. Lastly, GlycoNPs were functionalized with thiolated anti-cMyc siRNA through direct attachment of the thiol group to the gold core of the AuNP by establishing strong pseudo-covalent bonds Au-S (for further details on synthesis, functionalization and quantification methods for siRNA gold glycoNPs see the ESI $\dagger$ ).

\section{Triggering apoptotic pathways via glyconanoparticles}

The functionalized nanoparticles were administered in vitro to a luciferase-CMT/167 adenocarcinoma cancer cell line and in vivo via instillation directly into the lungs of cancer mice (B6 albino mice induced with luciferase-CMT/167 adenocarcinoma cells). Upon 48 hours of treatment with increasing concentrations (10, 100 and $200 \mu \mathrm{g} \mathrm{mL}^{-1}$ ) of PEGylated GlycoNPs (AuNP@) PEG@Glucose) and siRNA GlycoNPs (AuNP@PEG@Glucose@ siRNA) cells were assessed for the expression of proapoptotic proteins such as Fas/CD95 and caspases-3 and -9, as well as the siRNA target $c-M y c$ gene that also triggers apoptosis in association with tumour suppressors such as ARF and p53. ${ }^{22}$

Representative confocal images of luciferase-CMT/167 adenocarcinoma-loaded cells show that siRNA GlycoNPs accomplish high cellular uptake (3-fold) and trigger apoptosis by enhancing the expression of the Fas death receptor, as detected by a significant increase (2-fold) of Fas/CD95 fluorescence (Fig. 2A).

Glucose-surface functionalization has also been developed for siRNA GlycoNPs as a metabolic transporter to enhance the uptake of the therapeutic nanoparticles by cancer cells. In fact, tumour cells display a high rate of glucose uptake and glycolysis, since cell proliferation requires increased uptake of nutrients (e.g. glucose and glutamine). Cancer cells exhibit a high rate of glycolysis even in the presence of oxygen (aerobic glycolysis). ${ }^{23}$ The major function of aerobic glycolysis is to maintain high levels of glycolytic intermediates to support anabolic reactions in cells. This explains increased glucose metab-
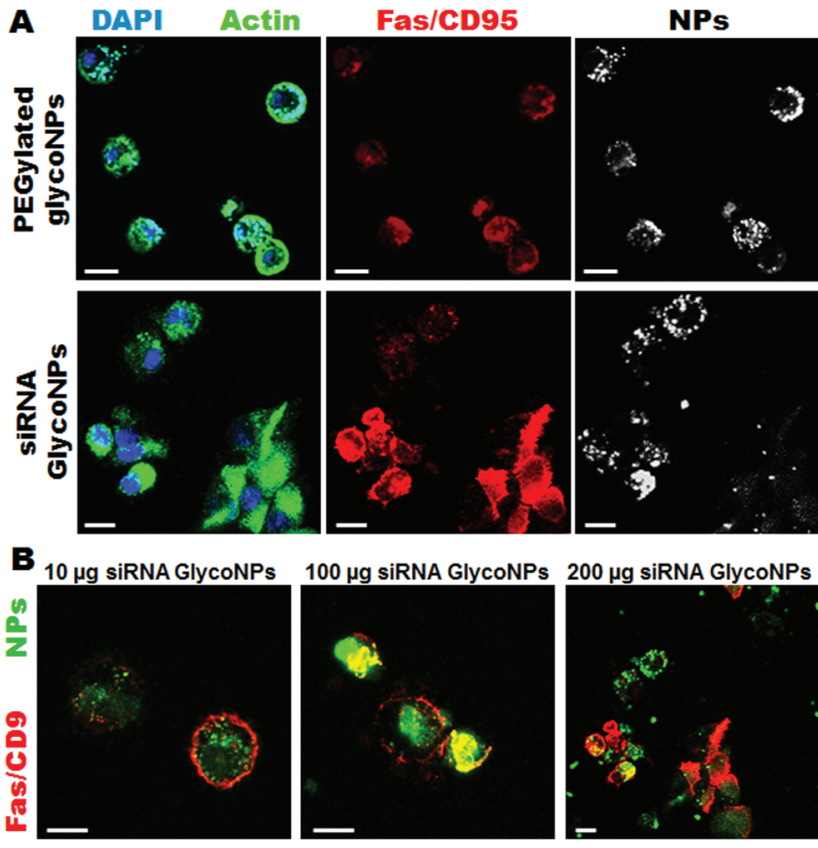

Fig. 2 Gold siRNA glyconanoparticles trigger apoptotic pathways via expression of the Fas cell death receptor. (A) Immunostaining of Fas/ CD95 in luciferase-CMT/167 adenocarcinoma cells treated with $200 \mu \mathrm{g}$ $\mathrm{mL}^{-1}$ of PEGylated glycoNPs and siRNA GlycoNPs for 48 hours. Cells stained for DAPI (nuclei) in blue, actin (phalloidin) in green, Fas/CD95 stained in red and NPs (conjugated streptavidin with Cy7-Allophycocyanin bound with biotin) in white (scale bars, $20 \mu \mathrm{m}$ ). (B) Immunostaining of Fas/CD95 in luciferase-CMT/167 adenocarcinoma cells treated with 10,100 and $200 \mu \mathrm{g} \mathrm{mL}^{-1}$ of siRNA GlycoNPs. Cells stained for Fas/CD95 in red and NPs (conjugated streptavidin with Cy7-Allophycocyanin bound with biotin) in green (scale bars, $20 \mu \mathrm{m}$ ). The results shown are representative for at least three independent experiments.

olism in proliferating cells. ${ }^{18}$ In addition to cell proliferation, increased glucose uptake may also be associated with mitochondria damage in cancer cells, or an adaptation to hypoxia environments within tumours or to mitochondria shutdown by cancer genes (e.g. $c-M y c)$ involved in the cell's apoptosis program. ${ }^{23,24}$ In fact, high glucose levels proved to be pro-apoptotic, increasing the sensitivity to apoptosis via Fas activation. ${ }^{14}$

Our results suggest that siRNA GlycoNPs activate apoptotic pathways by regulating cell death receptors and effective caspases, and the effect is dose dependent. Increasing concentrations $\left(10,100\right.$ and $\left.200 \mu \mathrm{g} \mathrm{mL}^{-1}\right)$ of siRNA GlycoNPs show enhancement in Fas expression in a dose dependent manner, as it can be seen by an increase of Fas/CD95 fluorescence (see Fig. 2B) 1.5-fold from 10 to $100 \mu \mathrm{g} \mathrm{mL}^{-1}$ and 3-fold from 100 to $200 \mu \mathrm{g} \mathrm{mL}^{-1}$ of NPs.

In order to corroborate these data and evaluate siRNA GlycoNPs' involvement in the activation of crucial proapoptotic proteins, expression of Fas, caspase- 3 and caspase- 9 was assessed by western blotting in the luciferase-CMT/167 adenocarcinoma cancer cell line. The expression of Fas, activated/ cleaved caspase- 3 and caspase- 9 proteins was significantly higher (5 fold) for siRNA GlycoNPs at 10, 100 and $200 \mu \mathrm{g} \mathrm{mL}^{-1}$ compared to PEGylated GlycoNPs (Fig. 3A). 
A
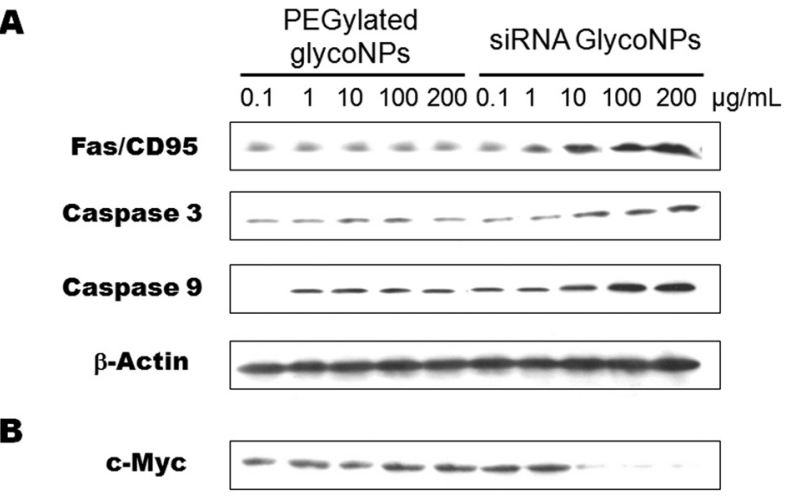

C
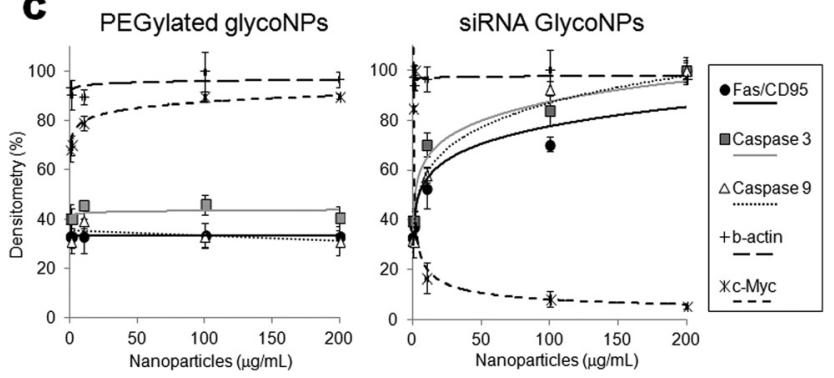

Fig. 3 Activation of the apoptotic pathway via siRNA GlycoNPs. Fas, Caspase 3, Caspase 9 (A) and C-Myc expression (B) in western blot after luciferase-CMT/167 adenocarcinoma cancer cells treated with increasing concentrations $\left(0.1,1,10,100\right.$ and $\left.200 \mu \mathrm{g} \mathrm{mL}^{-1}\right)$ of PEGylated GlycoNPs and siRNA GlycoNPs. (C) Comparative graph with the expression of Fas, and activated Caspases 3 and 9 and C-Myc after luciferase-CMT/167 adenocarcinoma cancer cells treated with PEGylated GlycoNPs and siRNA GlycoNPs. The results shown are representative for at least three independent experiments.

The expression of Fas and caspase- 3 and -9 from cells treated with $10 \mu \mathrm{g} \mathrm{mL}^{-1}$ of siRNA GlycoNPs indicates the strong activation of both intrinsic and extrinsic apoptotic pathways. Enhanced activation of caspase-9 by siRNA GlycoNPs can especially be observed at $200 \mu \mathrm{g} \mathrm{mL}^{-1}$ (see Fig. 2), which in turn may induce the activation of procaspase-3, which leads to the apoptosome formation. ${ }^{24}$ Once formed, the apoptosome can then recruit and activate the inactive pro-caspase-9, which can activate effector caspases and trigger a cascade of events leading to apoptosis. Finally, after the activation of caspase-9, the effector caspase-3 is activated and marks the endpoint of apoptosis. $^{24}$

This finding further supports the notion that siRNA GlycoNPs promote apoptosis within the luciferase-CMT/167 adenocarcinoma cancer cell line when exposed for 48 hours at a concentration of 100 and $200 \mu \mathrm{g} \mathrm{ml}^{-1}$ (Fig. 3A and C). PEGylated GlycoNPs showed no changes in expression of the assessed cell death receptor or caspases (Fig. 3A and C). Several studies were published in the last years reporting that some engineered AuNPs induce apoptosis in cells via caspase pathways. $^{25-30}$ Our platform comprises a new and smart siRNA gold glycoNP system capable of inducing apoptosis via hyperactivation of cell death receptors and caspases pathways.
RNAi-based GlycoNPs were also functionalized with a siRNA to mediate silencing of $c-M y c$ gene expression. $c-M y c$ is widely known as a crucial regulator of cell proliferation in normal and neoplastic cells, and recent reports strongly support a dual function model for $c-M y c$ as a co-ordinate activator of cell proliferation and apoptosis. ${ }^{28}$ Several studies provide evidence that $c-M y c$ function is closely related to apoptosis and that the induction or inhibition of apoptosis by this protein may depend on the level of expression or cell lineages used. $^{31}$ To assess whether anti-cMyc siRNA GlycoNPs attenuate MYC protein synthesis, its expression was also evaluated by western blotting in the luciferase-CMT/167 adenocarcinoma cancer cell line (Fig. 3B and C) after exposure to PEGylated GlycoNPs and siRNA GlycoNPs. Western blotting results clearly show that $c-M y c$ downregulation (14-fold) is attained with only 10 $\mu \mathrm{g} \mathrm{mL}^{-1}$ of siRNA GlycoNPs (Fig. 3B and C). PEGylated GlycoNPs show no silencing effect for all NP concentrations (Fig. 3B and C).

\section{In vivo RNAi triggering via siRNA glyconanoparticles}

For in vivo evaluation of the effect of GlycoNP treatment on $c-M y c$ expression in a mouse lung tumour model, $200 \mu \mathrm{g} \mathrm{mL}$ of PEGylated GlycoNPs and siRNA GlycoNPs were administered to mice previously inoculated with luciferase-CMT/167 adenocarcinoma cells. Four weeks after orthotropic lung cancer induction mice were treated by instillation of 0.3 pmol AuNPs (at weeks 12, 13, 14 and 15), respectively, in $50 \mu \mathrm{l}$ pyrogen-free distilled water, followed by $100 \mu \mathrm{l}$ of air. Fig. 4A shows Hematoxylin and Eosin staining (H\&E) and immunolocalisation of $c-M y c$ expression in mice lung tissues for sham, PEGylated GlycoNPs and siRNA GlycoNPs groups. The PEGylated GlycoNP treated group demonstrates a cancer lung tissue characterized by hypercellularity and thickened alveolar septa. Cancer cells have spread throughout the lung tissue, and large areas of deformed lung structures can be observed (Fig. 4A). Severe interstitial inflammatory cell infiltration is noted in the PEGylated GlycoNP treated group, with the predominance of perivascular and peribronchiolar edemas (Fig. 4A arrows). In the siRNA GlycoNP-treated group in contrast a significant decrease in the incidence and severity of tumour foci in the lungs can be observed, revealing the potency of siRNA GlycoNPs in reducing the tumour mass in the lung cancer mouse model. Only a few scattered cancer cells can get localized with recovered alveolar lung tissue. Concerning confocal images of lung tissue, Fig. 4B shows high expression of the MYC protein in the cytoplasm of tumour cells after exposure to PEGylated GlycoNPs whereas Fig. 4C illustrates tumour cells treated with siRNA GlycoNPs and shows downregulation of local MYC expression (6-fold), probably leading tumour cells to apoptosis, and consequently inhibiting cell proliferation. Moreover, cancer cells in lung tissue accumulate more siRNA GlycoNPs (2-fold) (Fig. 4C) than PEGylated GlycoNPs (Fig. 4B). Xing and co-workers also reported that glucose and antisense oligodeoxynucleotide-capped AuNPs showed significantly increased cellular uptake compared to neutral nanoparticles in breast cancer ${ }^{32}$ and prostate cancer cells. ${ }^{33}$ 


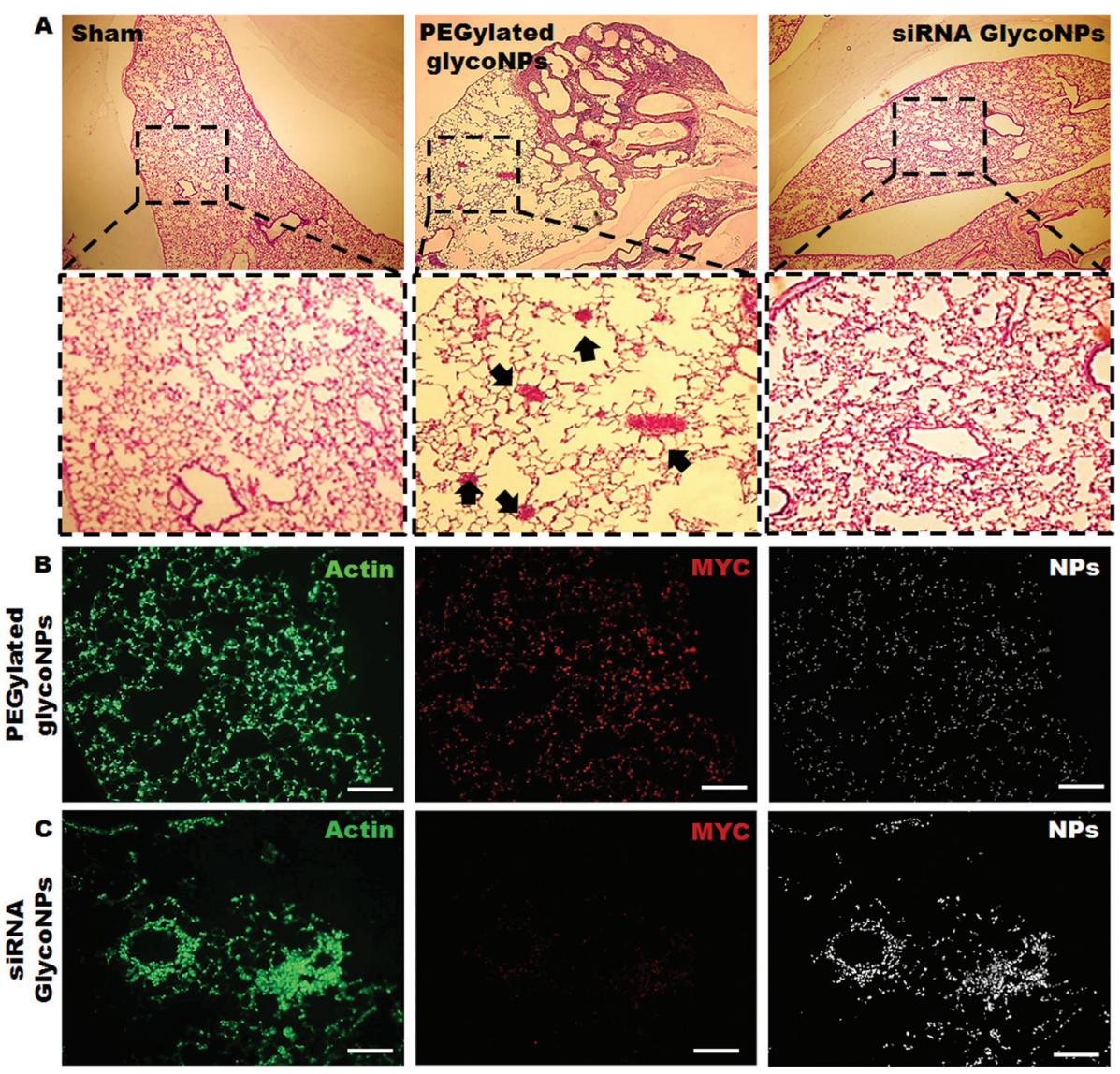

Fig. 4 RNAi triggering in mice. (A) Hematoxylin and eosin (H\&E) stains of sham (healthy mice without lung cancer induction of luciferase-CMT/167 adenocarcinoma cells), PEGylated GlycoNP and siRNA GlycoNP treated groups in lung tumor tissue. Severe interstitial inflammatory cell infiltration is noted, with the predominance of perivascular and peribronchiolar edemas (arrows) in PEGylated GlycoNPs only. Immunohistochemical images of lung tissue of PEGylated GlycoNP (B) and siRNA GlycoNP (C) treated groups. Cells in lung tissue stained for actin in green and MYC stained in red (scale bars, $100 \mu \mathrm{m}$ ).

Inflammatory response and in vivo glyconanoparticle biodistribution in mice

The inflammatory response of the assembled glyco-nanoconjugates was evaluated by bronchoalveolar lavage (BAL) cell analysis. Under steady state conditions, the most abundant cells retrieved in the BAL fluid are the resident alveolar macrophages that line the alveolar space (about $98 \%$ of BAL cells), and under inflammatory conditions infiltrating leukocytes such as lymphocytes and neutrophils. Consequently, evaluation of the number of BAL macrophages, lymphocytes and neutrophils was used to characterize the inflammatory response (Fig. 5) upon treatment with PEGylated GlycoNPs and siRNA GlycoNPs in cancer mice for 1, 3 and 14 days. Fig. 5A shows representative microscopy images of BAL cells at days 1 and 14 for $200 \mu \mathrm{g} \mathrm{mL} \mathrm{L}^{-1}$ of PEGylated GlycoNPs and siRNA GlycoNPs. Fig. 5B shows evaluation of the number of macrophages, lymphocytes and neutrophils in cancer mice for 1,3 and 14 days after PEGylated GlycoNP and siRNA GlycoNP instillation. No multinucleated macrophages, indicators of a foreign body response, are found under any conditions. As expected, ${ }^{13}$ the sham treated group (healthy mice without lung cancer induction of luciferase-CMT/167 adenocarcinoma cells) reveals intact and normal alveolar macrophages (Fig. 5A). Inflammatory cells infiltration is noted by increasing numbers of neutrophils in the siRNA GlycoNP $\left(200 \mu \mathrm{g} \mathrm{mL} \mathrm{m}^{-1}\right)$ treated groups on days 1 and 14 (Fig. 5A). PEGylated and siRNA GlycoNPs show the same pattern and number of macrophages as well as neutrophils and lymphocytes (Fig. 5B). The increased number of neutrophils with $200 \mu \mathrm{g} \mathrm{mL} \mathrm{mL}^{-1}$ of PEGylated and siRNA GlycoNPs on day 1 indicates a moderate, acute and over time rapidly declining inflammatory response, resulting in baseline levels (sham) and thus negligible neutrophil numbers at days 3 and 14 after treatment (Fig. 5B). No significant changes have been observed for BAL lymphocyte numbers over the period of investigation, pointing to the generally described innate, acute inflammatory response to pulmonary deposited materials. ${ }^{34,35}$ No significant changes in the number of macrophages and neutrophils between PEGylated GlycoNPs and siRNA GlycoNPs are observed (Fig. 5B).

In order to analyse the tumour size, nanoparticle uptake and biodistribution in the whole body of mice but predominantly in lung tumour tissue, tomography bioluminescence 


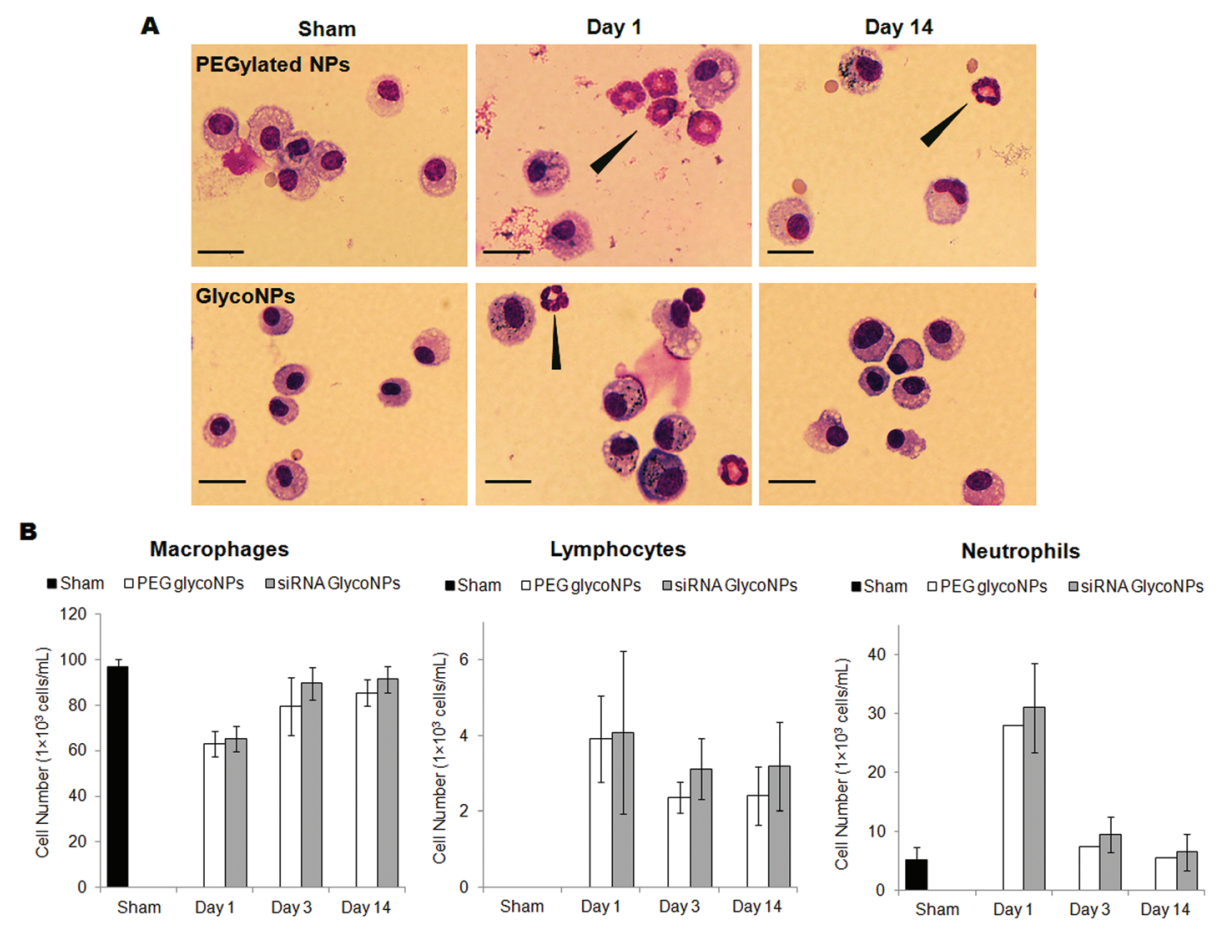

Fig. 5 Inflammatory response in bronchoalveolar lavage (BAL) cells in mice during 1, 3 and 14 days of exposure to gold GlycoNPs. (A) Representative microscopy images of BAL cells (scale bars, $20 \mu \mathrm{m}$ ) at days 1 and 14 for $200 \mu \mathrm{g} \mathrm{mL} \mathrm{L}^{-1}$ of PEGylated GlycoNPs and siRNA GlycoNPs. Black arrows indicate neutrophils. (B) Evaluation of the number of macrophages, lymphocytes and neutrophils was used to detect the inflammatory response in the lungs during 1, 3 and 14 days after PEGylated GlycoNP and siRNA GlycoNP instillation. Sham groups correspond to healthy mice without lung cancer induction and any nanoparticle treatment. Error bars indicate \pm s.d. of $n=6$ mice.

imaging was carried out in the mouse cancer model (B6 albino female mice injected with luciferase-CMT/167 adenocarcinoma cells) treated with $200 \mu \mathrm{g} \mathrm{mL}^{-1}$ of PEGylated GlycoNPs and siRNA GlycoNPs (Fig. 6). Representative whole body images of the individual mice from each treated group ( $n=8$ animals) shown at a fixed photon flux scale illustrate the luciferase activity (Fig. 6A). Detection of epi-fluorescence (Fig. 6B) further allowed localization of nanoparticles conjugated to streptavidin with Cy7-Allophycocyanin (Cy7APC, BD Pharmingen $^{\mathrm{TM}}$ ) with an excitation wavelength of $642 \mathrm{~nm}$. Fig. 6C and D show excised organs to evaluate the lung tumour size via the luminescence of luciferase and Fig. $6 \mathrm{E}$ and $\mathrm{F}$ show nanoparticle distribution in mice organs via the measurement of epi-fluorescence of nanoparticles conjugated to streptavidin with Cy7-Allophycocyanin $($ Exc $=642 \mathrm{~nm})$.

The tomography images clearly depict that siRNA GlycoNPs achieved an increased targeting toward lung cancer cells when compared to PEGylated GlycoNP treatment, which shows no signal-accumulation in the respective lung tissue, for the same scale of epi-fluorescence. Once the lung is characterized by high expression of $c-M y c$, siRNA anti-cMyc targeting GlycoNPs seem to accumulate in the tissue more effectively and for a longer period of time than observed for PEGylated GlycoNPs only. Moreover, treatment of the murine lung cancer model with GlycoNPs leads to successful tumour size reduction $(\sim 80 \%)$ as depicted by the decrease in the reporter lumines- cence signal (Fig. 6A and C), when compared to the PEGylated GlycoNPs.

\section{Experimental}

\section{Synthesis of PEGylated and siRNA GlycoNPs}

A full description of synthesis and characterization methods of PEGylated GlycoNPs and siRNA GlycoNPs can be found in the ESI. $\dagger$

\section{Immunostaining of luciferase-CMT/167 adenocarcinoma cells}

Cells were fixed with $-20{ }^{\circ} \mathrm{C}$ methanol for $10 \mathrm{~min}$ and with $-20{ }^{\circ} \mathrm{C}$ acetone for $1 \mathrm{~min}$ on cover slips. The cover slips were washed twice in PBS, and then blocked with PBS containing $0.1 \%$ BSA for $10 \mathrm{~min}$ at room temperature followed by draining. The cell-side-up of the glass slide was incubated with antirabbit Fas (Santa Cruz Biotechnology) in PBS containing 1\% BSA for $60 \mathrm{~min}$, and was washed three times in PBS. The samples were incubated with Alexa 546 anti-rabbit or FITC 488 anti-rabbit as the secondary antibody and phalloidin (Invitrogen), at the recommended dilution, in PBS containing 1\% $\mathrm{BSA}$, for $30 \mathrm{~min}$, and then were washed three times in PBS. After DAPI staining, one drop of aqueous mounting medium was added on the cover slip and inverted carefully on the glass slide. The images were acquired on a Zeiss confocal micro- 


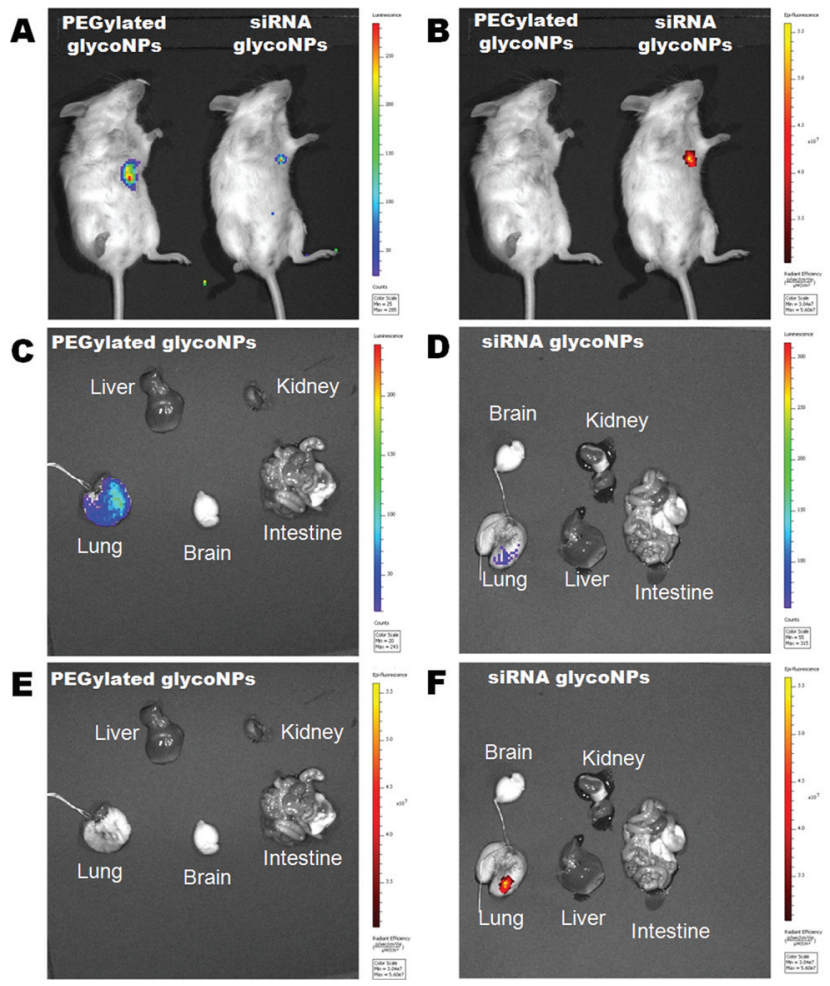

Fig. 6 GlycoNP uptake, whole body biodistribution and tumour size assessment in mice. Tomography imaging of B6 albino female mice injected with luciferase-CMT/167 adenocarcinoma cells $(n=8$ animals per treated group) treated with $200 \mu \mathrm{g} \mathrm{mL}^{-1}$ of PEGylated GlycoNPs and siRNA GlycoNPs. Representative imaging of individual mice from each treatment group ( $n=8$ animals) is shown, with the same scale of photon flux indicating luciferase activity (A) and epi-fluorescence (B) indicating nanoparticles conjugated to streptavidin with Cy7-Allophycocyanin (Cy7APC, BD Pharmingen ${ }^{\mathrm{TM}}$ ) with an excitation wavelength of $642 \mathrm{~nm}$. (A, B) Luminescence and epi-fluorescence images of lung cancer mice treated with $200 \mu \mathrm{g} \mathrm{mL} \mathrm{m}^{-1}$ of PEGylated GlycoNPs and siRNA GlycoNPs. Excised organs to evaluate the lung tumour size via the luminescence of luciferase in mice treated with PEGylated AuNPs (C) and gold GlycoNPs (D). Nanoparticle distribution in different organs treated with PEGylated GlycoNPs (E) and siRNA GlycoNPs (F) via the measurement of epi-fluorescence of nanoparticles conjugated to streptavidin with Cy7-Allophycocyanin.

scope and the fluorescence density per cell was analysed by using ImageJ.

\section{Western blot of Fas/CD95, caspases 3 and 9 expression}

Protein extract was boiled in sample buffer for $3 \mathrm{~min}$ and loaded onto $12 \%$ SDS-polyacrylamide gel. Details of the western immunoblotting procedure have been described elsewhere. ${ }^{36}$ The antibodies used in this experiment were as follows: anti-rabbit Fas (Santa Cruz Biotechnology, 1:500 dilution), anti-rabbit caspase3 (cleaved) (Invitrogen, 1:500 dilution), anti-rabbit polyclonal caspase9 (cleaved) (Abcam, $1: 1000$ dilution), and anti-rabbit IgG horseradish peroxidase (Amersham-Pharmacia Biotech, 50 000:1 dilution). The blot was visualized with an ECL kit (Amersham-Pharmacia Biotech).

\section{In vivo targeting of PEGylated glycoNPs and siRNA GlycoNPs}

Prior to glycoNP instillation, B6 albino mice (B6N-Tyr ${ }^{\mathrm{c}} /$ BrdCrCrl, Charles River, France, induced with luciferase-CMT/ 167 adenocarcinoma cells) were anesthetized by intraperitoneal injection of a mixture of medetomidine $(0.5 \mathrm{mg}$ per $\mathrm{kg}$ body mass), midazolam (5.0 $\mathrm{mg}$ per $\mathrm{kg}$ body mass) and fentanyl (0.05 mg per $\mathrm{kg}$ body mass). The animals were then intubated using a nonsurgical technique. Using a cannula inserted $10 \mathrm{~mm}$ into the trachea, a suspension containing $1 \times 10^{5}$ cancer cells or $200 \mu \mathrm{g} \mathrm{mL} \mathrm{mL}^{-1}$ of PEGylated GlycoNPs and siRNA GlycoNPs in $50 \mu \mathrm{l}$ pyrogen-free distilled water was instilled, followed by $100 \mu \mathrm{l}$ air. After instillation the animals were anaesthesised by subcutaneous injection of a mixture of atipamezole (2.5 mg per $\mathrm{kg}$ body mass), flumazenil (0.5 mg per $\mathrm{kg}$ body mass) and naloxone (1.2 $\mathrm{mg}$ per $\mathrm{kg}$ body mass) and to control awakening of the mice. Animal experiments were carried out according to the German law of protection of animal life and were approved by an external review committee for laboratory animal care (animal approval number: 55.2-1-542532-20-11). After 72 hours mice lungs were dissected and prepared for Hematoxylin and Eosin staining (H\&E) and for immunolocalisation by a standard immunohistochemical procedure to verify $c-M y c$ expression and the distribution of NPs by tissue sections.

\section{Molecular analyses of MYC expression in mice}

The lung cancer tissues (from B6 albino mice induced with luciferase-CMT/167 adenocarcinoma cells) were embedded and sliced into $3 \mu \mathrm{m}$ sections. Standard Hematoxylin and Eosin (H\&E) staining was employed for cancer cell morphology observation. For immunolocalisation, standard immunohistochemical staining methods were employed: the tissue slide was incubated with anti-mouse $c-M y c$ and anti-rabbit caspase-3 $(1: 1000)$ in PBS containing $1 \%$ BSA for $60 \mathrm{~min}$, and was washed three times in Tris-buffered saline (TBS). The slide was incubated with $560 \mathrm{~nm}$ anti-mouse and FITC anti-rabbit as the secondary antibody, at the recommended dilution, in TBS containing 1\% BSA, for $30 \mathrm{~min}$, and then was washed three times in PBS. The slides were mounted with $50 \mu \mathrm{l}$ of mounting medium. The images were acquired by Zeiss confocal microscopy and the fluorescence density per cell was analysed by using ImageJ.

\section{Preparation of BAL cells/fluid for evaluation of the inflammatory response}

BAL (bronchoalveolar lavage cell) fluid was obtained by injecting 4 times and recovering two $0.5 \mathrm{~mL}$ aliquots of PBS via a tracheal cannula. Cells recovered with the lavage fluid (lymphocytes and neutrophils accompanying the predominant population of alveolar macrophages) were determined utilizing light microscopic cell differentiation, counting 200 cells per cytospin preparation. Details of the bronchoalveolar lavage procedure were described elsewhere. ${ }^{37}$ Mice were exposed to $200 \mu \mathrm{g} \mathrm{mL} \mathrm{L}^{-1}$ of PEGylated GlycoNPs or siRNA GlycoNPs. 


\section{In vivo bioluminescence imaging}

Stable clones of CMT64/61 cells, originally derived from spontaneous lung adenocarcinoma of a $\mathrm{C} 57 \mathrm{Bl} / \mathrm{ICRF}$ mouse, ${ }^{32}$ were generated by transfection with pGL3-Control vector (Promega $\mathrm{GmbH}$ ), and co-transfection for selection with a linear Hygromycin resistance marker (Clontech). A stable clone (CMT/167luc) expressing high levels of firefly luciferase, constitutively driven by the SV40 promoter and enhancer, was instilled into female C57BL/6 albino mice. Prior to instillation, the mice were anesthetized by intraperitoneal injection of a mixture of medetomidine ( $0.5 \mathrm{mg}$ per $\mathrm{kg}$ body mass), midazolam $(5.0 \mathrm{mg}$ per $\mathrm{kg}$ body mass) and fentanyl (0.05 $\mathrm{mg}$ per $\mathrm{kg}$ body mass). The animals were then intubated using a nonsurgical technique. Using a cannula inserted $10 \mathrm{~mm}$ into the trachea, a suspension containing $1 \times 10^{5} \mathrm{CMT} / 167$-luc cells in $50 \mu \mathrm{l}$ pyrogenfree distilled water was instilled, followed by $100 \mu \mathrm{l}$ of air, at week 8. Four weeks after orthotropic lung cancer induction the mice were treated by instillation of $0.3 \mathrm{pmol}$ AuNP (at weeks 12, 13, 14 and 15), respectively, in $50 \mu$ l pyrogen-free distilled water, followed by $100 \mu \mathrm{l}$ of air. After instillation animals were antagonized by subcutaneous injection of a mixture of atipamezole (2.5 mg per $\mathrm{kg}$ body mass), flumazenil ( $0.5 \mathrm{mg}$ per $\mathrm{kg}$ body mass) and naloxone (1.2 $\mathrm{mg}$ per $\mathrm{kg}$ body mass). Luciferase expression was monitored applying the IVIS® imaging system (Lumina, PerkinElmer) from mice bearing tumours from luciferase-CMT/167 cells ( $n=8$ animals per treated group).

\section{Conclusions}

Several studies have been published in the last few years reporting that some engineered gold nanoparticles induce apoptosis in cells via caspase pathways. Herein, we present for the first time a new and smart RNAi-based gold glyconanoparticle system capable of inducing apoptosis via hyperactivation of cell death receptors and caspase pathways. In summary, we demonstrate that siRNA GlycoNPs have remarkable potential to trigger apoptosis via the enhancement of cell death receptors and activation of caspases. Moreover, our results confirm that sham, PEGylated GlycoNP and siRNA GlycoNP treated groups cause the same inflammatory response, proving that siRNA GlycoNPs trigger apoptotic pathways via expression of cell death receptors and effective caspases in a specific way, independent of the inflammatory response. Consequently, the switching on of the apoptotic pathways is not caused by any toxic and/or adverse side effects of the exposure to the NPs in mice. Most importantly, pulmonary delivery of siRNA GlycoNPs leads to a $\sim 80 \%$ reduction in the tumour size via in vivo RNAi in tumour tissue by targeting $c-M y c$ gene expression.

\section{Acknowledgements}

The authors thank the ERANET-NANOSCIERA NANOTRUCK project for financial support. JMF thanks Fondo Social Europeo for financial support.

\section{Notes and references}

1 C. M. Dong, Glyconanoparticles for biomedical applications, Comb. Chem. High Throughput Screening, 2011, 14, 173-181.

2 J. M. de la Fuente and S. Penades, Understanding carbohydrate-carbohydrate interactions by means of glyconanotechnology, Glycoconjugate J., 2004, 21, 149-163.

3 J. M. de la Fuente, A. G. Barrientos, T. C. Rojas, J. Rojo, J. Canada, A. Fernandez and S. Penades, Gold glyconanoparticles as water-soluble polyvalent models to study carbohydrate interactions, Angew. Chem., Int. Ed., 2001, 40, 22582261.

4 A. G. Barrientos, J. M. de la Fuente, T. C. Rojas, A. Fernandez and S. Penades, Gold glyconanoparticles: Synthetic polyvalent ligands mimicking glycocalyx-like surfaces as tools for glycobiological studies, Chem. - Eur. J., 2003, 9, 1909-1921.

5 J. M. de la Fuente and S. Penades, Glyconanoparticles: types, synthesis and applications in glycoscience, biomedicine and material science, Biochim. Biophys. Acta, 2006, 1760, 636-651.

6 J. M. de la Fuente, D. Alcantara, P. Eaton, P. Crespo, T. C. Rojas, A. Fernandez, A. Hernando and S. Penades, Gold and gold-iron oxide magnetic glyconanoparticles: synthesis, characterization and magnetic properties, J. Phys. Chem. B, 2006, 110, 13021-13028.

7 J. Rojo, V. Diaz, J. M. de la Fuente, I. Segura, A. G. Barrientos, H. H. Riese, A. Bernade and S. Penades, Gold glyconanoparticles as new tools in antiadhesive therapy, ChemBioChem, 2004, 5, 291-297.

8 J. M. de la Fuente, P. Eaton, A. G. Barrientos, M. Menendez and S. Penades, Thermodynamic evidence for $\mathrm{Ca} 2+-$ mediated self-aggregation of Lewis $\mathrm{X}$ gold glyconanoparticles. A model for cell adhesion via carbohydrate-carbohydrate interaction, J. Am. Chem. Soc., 2005, 127, 61926197.

9 M. Reynolds, M. Marradi, A. Imberty, S. Penades and S. Perez, Multivalent Gold Glycoclusters: High Affinity Molecular Recognition by Bacterial Lectin PA-IL, Chem. Eur. J., 2012, 18, 4264-4273.

10 C. L. Schofield, A. H. Haines, R. A. Field and D. A. Russell, Silver and gold glyconanoparticles for colorimetric bioassays, Langmuir, 2006, 22, 6707-6711.

11 J. Conde, A. Ambrosone, V. Sanz, Y. Hernandez, V. Marchesano, F. Tian, H. Child, C. C. Berry, M. R. Ibarra, P. V. Baptista, C. Tortiglione and J. M. de la Fuente, Design of Multifunctional Gold Nanoparticles for In Vitro and In Vivo Gene Silencing, ACS Nano, 2012, 6, 8316-8324.

12 V. Sanz, J. Conde, Y. Hernandez, P. V. Baptista, M. R. Ibarra and J. M. de la Fuente, Effect of PEG biofunctional spacers and TAT peptide on dsRNA loading on gold nanoparticles, J. Nanopart. Res., 2012, 14.

13 J. Conde, F. Tian, Y. Hernandez, C. Bao, D. Cui, K. P. Janssen, M. R. Ibarra, P. V. Baptista, T. Stoeger and J. M. de la Fuente, In vivo tumor targeting via nanoparticle- 
mediated therapeutic siRNA coupled to inflammatory response in lung cancer mouse models, Biomaterials, 2013, 34, 7744-7753.

14 K. Maedler, G. A. Spinas, R. Lehmann, P. Sergeev, M. Weber, A. Fontana, N. Kaiser and M. Y. Donath, Glucose induces beta-cell apoptosis via upregulation of the Fas receptor in human islets, Diabetes, 2001, 50, 1683-1690.

15 R. W. Y. Yeo, K. Y. Yang, G. D. Li and S. K. Lim, High Glucose Predisposes Gene Expression and ERK Phosphorylation to Apoptosis and Impaired Glucose-Stimulated Insulin Secretion via the Cytoskeleton, PLoS One, 2012, 7.

16 M. Lorenzi, D. F. Montisano, S. Toledo and A. Barrieux, High Glucose Induces DNA Damage in Cultured HumanEndothelial Cells, J. Clin. Invest., 1986, 77, 322-325.

17 M. Lorenzi, E. Cagliero and S. Toledo, Glucose Toxicity for Human-Endothelial Cells in Culture - Delayed Replication, Disturbed Cell-Cycle, and Accelerated Death, Diabetes, 1985, 34, 621-627.

18 M. Lopez-Lazaro, The warburg effect: why and how do cancer cells activate glycolysis in the presence of oxygen?, Anti-Cancer Agents Med. Chem., 2008, 8, 305-312.

19 J. Turkevich, P. C. Stevenson and J. Hillier, A study of the nucleation and growth processes in the synthesis of colloidal gold, Discuss. Faraday Soc., 1951, 11, 55-75.

20 G. Frens, Controlled Nucleation for the Regulation of the Particle Size in Monodisperse Gold Suspensions, Nature Phys. Sci., 1973, 241, 20-22.

$21 \mathrm{X}$. Zhang and V. K. Yadavalli, Functionalized selfassembled monolayers for measuring single molecule lectin carbohydrate interactions, Anal. Chim. Acta, 2009, 649, 1-7.

22 D. N. Boone, Y. Qi, Z. L. Li and S. R. Hann, Egr1 mediates p53-independent c-Myc-induced apoptosis via a noncanonical ARF-dependent transcriptional mechanism, Proc. Natl. Acad. Sci. U. S. A., 2011, 108, 632-637.

23 S. Y. Lunt and M. G. Vander Heiden, Aerobic Glycolysis: Meeting the Metabolic Requirements of Cell Proliferation, Annu. Rev. Cell Dev. Biol., 2011, 27, 441-464.

24 S. Elmore, Apoptosis: A review of programmed cell death, Toxicol. Pathol., 2007, 35, 495-516.

25 J. C. Mohan, G. Praveen, K. P. Chennazhi, R. Jayakumar and S. V. Nair, Functionalised gold nanoparticles for selective induction of in vitro apoptosis among human cancer cell lines, J. Exp. Nanosci., 2013, 8, 32-45.

26 H. K. Patra, S. Banerjee, U. Chaudhuri, P. Lahiri and A. K. Dasgupta, Cell selective response to gold nanoparticles, Nanomedicine-Nanotechnology Biology and Medicine, 2007, 3, 111-119.
27 W. Gao, K. H. Xu, L. F. Ji and B. Tang, Effect of gold nanoparticles on glutathione depletion-induced hydrogen peroxide generation and apoptosis in HL7702 cells, Toxicol. Lett., 2011, 205, 86-95.

28 P. Mukherjee, R. Bhattacharya, N. Bone, Y. K. Lee, C. R. Patra, S. Wang, L. Lu, C. Secreto, P. C. Banerjee, M. J. Yaszemski, N. E. Kay and D. Mukhopadhyay, Potential therapeutic application of gold nanoparticles in B-chronic lymphocytic leukemia (BCLL): enhancing apoptosis, J. Nanobiotechnol., 2007, 5, 4.

29 B. Kang, M. A. Mackey and M. A. El-Sayed, Nuclear Targeting of Gold Nanoparticles in Cancer Cells Induces DNA Damage, Causing Cytokinesis Arrest and Apoptosis, J. Am. Chem. Soc., 2010, 132, 1517-1519.

30 I. C. Sun, S. Lee, H. Koo, I. C. Kwon, K. Choi, C. H. Ahn and K. Kim, Caspase Sensitive Gold Nanoparticle for Apoptosis Imaging in Live Cells, Bioconjugate Chem., 2010, 21, 1939-1942.

31 G. C. Prendergast, Mechanisms of apoptosis by c-Myc, Oncogene, 1999, 18, 2967-2987.

32 T. Kong, J. Zeng, X. Wang, X. Yang, J. Yang, S. McQuarrie, A. McEwan, W. Roa, J. Chen and J. Z. Xing, Enhancement of radiation cytotoxicity in breast-cancer cells by localized attachment of gold nanoparticles, Small, 2009, 4, 15371543.

33 W. Roa, X. Zhang, L. Guo, A. Shaw, X. Hu, Y. Xiong, S. Gulavita, S. Patel, X. Sun, J. Chen, R. Moore and J. Z. Xing, Gold nanoparticle sensitize radiotherapy of prostate cancer cells by regulation of the cell cycle, Nanotechnology, 2009, 20, 375101.

34 A. Beyerle, A. Braun, A. Banerjee, N. Ercal, O. Eickelberg, T. H. Kissel and T. Stoeger, Inflammatory responses to pulmonary application of PEI-based siRNA nanocarriers in mice, Biomaterials, 2011, 32, 8694-8701.

35 K. Ganguly, S. Upadhyay, M. Irmler, S. Takenaka, K. Pukelsheim, J. Beckers, E. Hamelmann, H. Schulz and T. Stoeger, Pathway focused protein profiling indicates differential function for IL-1B, -18 and VEGF during initiation and resolution of lung inflammation evoked by carbon nanoparticle exposure in mice, Part. Fibre Toxicol., 2009, 6, 31.

36 J. G. Steele, C. Rowlatt, J. K. Sandall and L. M. Franks, CellSurface Properties of High-Metastatic and Low-Metastatic Cell-Lines Selected from A Spontaneous Mouse Lung-Carcinoma, Int. J. Cancer, 1983, 32, 769-779.

37 F. R. Tian, T. Nakahara, M. Yoshida, N. Honda, H. Hirose and J. Miyakoshi, Exposure to power frequency magnetic fields suppresses X-ray-induced apoptosis transiently in Ku80-deficient xrs5 cells, Biochem. Biophys. Res. Commun., 2002, 292, 355-361. 